\title{
Facteurs génétiques et immunologiques déterminant la résistance à la bilharziose en région d'endémie
}

\section{Alain Dessein Pascal Rihet Christian Demeure Patricia Couissinier Olivia Bacellar Edgar M. Carvalho Sybille Kohlstaedt Helia Dessein Antonio Souza Aluizio Prata Veronica Goudot Alain Bourgois Laurent Abel}

Dans les régions de bilharziose endémique, les formes cliniques graves se rencontrent principalement chez les individus les plus infectés. Les niveaux d'infection sont en grande partie fonction (inverse) des capacités de résistance des sujets qui ont des contatcts avec les eaux infectées par Schistosoma mansoni ; ces capacités semblent dépendre des effets d'un gène co-dominant majeur. Les facteurs écologiques et comportementaux ont, quant à eux, une influence mesurable, mais moindre, sur les niveaux d'infection. $L$ 'acquisition de la résistance est un processus lent qui se prolonge jusqu'à l'adolescence, qui est altéré dans les zones à haute transmission chez les sujets (adolescents) les plus exposés, et qui est associée au développement d'une immunité relayée par les IgE et dont les effets protecteurs sont contrebalancés par l'action négative des IgG4. L'augmentation des IgG4 spécifiques chez les adolescents très exposés, expliquerait la plus grande susceptibilité de ceux-ci à l'infection. Ces observations épidémiologiques et immunologiques plaident fortement pour un contrôle de cette endémie par la vaccination. Certaines enzymes parasitaires semblent être des cibles vaccinales prometteuses.

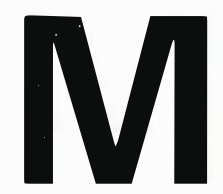

algré les progrès importants qui ont été effectués dans la prévention et le traitement des formes cliniques graves de bilharziose, aucune méthode ne permet actuellement de contrôler durablement ces endémies et certaines d'entre elles, telle la bilharziose à $S$. mansoni (figure 1, p. 110), sont en extension [1]. Le contrôle des bilharzioses, comme celui de la plupart des endémies parasitaires, doit se fonder sur une bonne connaissance des facteurs qui déterminent les hauts niveaux d'infection et le développement des formes cliniques graves; morbidité et hauts niveaux d'infection étant d'ailleurs étroitement liés. Parmi les facteurs capables d'influencer infection et maladie en zone de bilharziose endémique, doivent être distingués les facteurs de milieu soit liés à l'environnement, par exemple la densité de mollusques infectés ou la température de l'eau, soit d'origine comportementale, par exemple la fréquence des bains dans une rivière infectée - et les facteurs de susceptibilité/résistance qui traduisent les capacités de résistance intrinsèques à chaque individu et qui peuvent dépendre, par exemple, d'une immunité innée ou acquise.

Ces facteurs peuvent paraître nombreux et variés et leurs effets diffici-
E.M. Carvalho : professeur associé de medecine. ral da Bahia, Salvador, Brésil. 
les à évaluer ; en fait, seuls ceux des facteurs qui ont un effet limitant sur le phénotype sur lequel on veut agir, sont à prendre en compte dans les programmes de contrôle. L'importance de certains facteurs, tels le niveau d'exposition au parasite, l'âge ou les capacités de résistance, avait été notée dans le passé, mais leurs rôle et poids respectifs n'avaient pas été évalués en l'absence de méthodes quantitatives de mesure et à cause de difficultés dues à l'interdépendance de certains de ces facteurs. Cette évaluation est maintenant possible en utilisant les méthodes d'analyse statistique multivariée et, pour les études génétiques, d'analyse de ségrégation, à la condition que les paramètres de l'infection soient quantifiés avec un maximum d'objectivité.

En collaboration avec nos collègues brésiliens, nous avons effectué une telle étude dont les principales observations sont résumées ci-dessous.

\section{Importance des facteurs de milieu dans l'infection humaine par S. mansoni}

Cette étude a été effectuée dans un village du Nord-Est brésilien où la bilharziose est la seule endémie parasitaire ayant des conséquences médicales majeures. Le village est traversé par une rivière qui est peuplée de nombreux mollusques dont certains sont infectés par Schistosoma mansoni; comme la rivière est la principale source d'eau pour les usages domestiques et pour l'irrigation, laquelle est obligatoire sous ce climat sec et aride, pratiquement toute la population est infectée et certains individus ont un niveau d'infection très élevé. Le groupe du Dr Prata avait, durant plusieurs années, tenté de contrôler la transmission du parasite dans ce village. La destruction du mollusque vecteur par épandage de mollusquicides avait eu peu d'effets durables sur la transmission, car la population de mollusques se reconstituait quelques mois après l'application de mollusquicides ; le déparasitage, par chimiothérapie des sujets infectés, combiné à l'application de mollusquicides avait réduit considérablement les niveaux d'infection, mais les habitants du village s'étaient réinfectés dans les mois suivant le traitement et, $m / s n^{\circ} 2$, vol. 8 , féurier 92 trois ans après le traitement, les niveaux d'infection étaient comparables à ceux observés avant traitement.

Nous avons tout d'abord évalué soigneusement le niveau d'exposition de chacun des individus étudiés. Ce travail a été facilité par le fait que tous fréquentaient les mêmes points d'eau ; ces endroits ont fait l'objet d'une surveillance étroite et constante pendant toute la durée de l'étude, ct les individus ayant des contacts avec les points d'eau, la nature de leurs contacts (bains, irrigation, lavage de vaisselle, lessive, etc.) et la durée de ceux-ci ont été notés. Ces données ont fourni une évaluation de l'importance des contacts avec la rivière de chacun des individus participant à l'étude. Cette évaluation fut grandement facilitée par la coopération de la population à laquelle les raisons de ce travail avaient été expliquées. Ces observations permirent d'évaluer l'influence du niveau d'exposition sur la charge parasitaire, laquelle était mesurée par comptage des œufs du parasite dans les selles. Les résultats $[2,3]$ confirmèrent que les niveaux d'infection sont effectivement fonction des niveaux d'exposition; toutefois, une grande partie des variations inter-individuelles des niveaux d'infection ne purent être imputées à des différences d'exposition. Les niveaux d'infection étaicnt égalcment très variables suivant l'âge des sujets ; en particulicr, les individus les plus infectés étaient, cn général, des enfants de 8 à 12 ans. Ce phénomène avait été noté dans plusieurs études et avait été généralement attribué à un haut niveau d'exposition de ces enfants. Une analyse multivariée des résultats [3], prenant en compte les différences d'exposition entre les classes d'âge, montra que cettc conclusion n'était pas cxacte et qu'une partie seulement des variations du niveau d'infection avec l'âge était due à des différences d'exposition. Ce résultat est illustré par la figure 2, p. 111 qui montre, pour chaque classe d'âge et pour chaque sexc, les différences d'exposition (2B), les niveaux d'infection avant (2A) et après $(2 C)$ prise en compte des différences d'exposition entre classes d'âge. Cc résultat, également obtenu dans deux autres études réalisécs sur des populations du Kenya [4] et de
Gambie [5], cxplique unc propriété communc aux bilharzioses et très probablement à plusicurs autres helminthiases telles les anguilluloses pour lesquelles les courbes infection/âgc ressemblent à celles des bilharzioses $[6,7]$. Un tcl résultat indique que lc dévcloppement complet de la résistance aux schistosomes (ct sans doute à d'autres helminthiases) suit un lent processus de maturation/différenciation. Comme cc phénomènc a été obscrvé également chez des adultes réccmment installés en zone d'cndémic [8], il cst possible que le développement lent de la résistance ne dépende pas de l'âge mais requicrt plutôt unc exposition prolongéc au parasitc et de multiples réinfections. Il cst probable aussi qu'au moins deux phénomènes concourent à l'acquisition progressive de la résistance : (a) une lentc maturation de ccrtains mécanismes immunologiques et (b) l'altération de ce proccssus dans certaines conditions d'exposition très élevéc telles cclles obscrvécs chcz. les jeuncs de 8 à 12 ans. Ccttc altération cst suggérée par les donnécs de réinfection après traitement qui indiquent unc susccptibilité anormalement élevéc chez les sujets appartcnant aux classes très exposées (C.D. Demcurc et al., soumis pour publication) ct cst corroboréc par la misc en évidence de factcurs immunologiques capables d'interférer avec les mécanismes de résistance $\{9,10\}$.

\section{Les niveaux de résistance sont en grande partie déterminés génétiquement}

Bicn que les effets des nivcaux d'cxposition ct de l'âge (ou du temps d'cxposition) aicnt été nets, ils n'expliquaicnt que 20 à $25 \%$ de la variance des niveaux d'infection ct, de toutc évidence, d'autres facteurs étaient impliqués.

Nous avions noté que les sujets très infectés étaicnt groupés dans ccrtaincs familles et non pas distribués de manière aléatoirc dans la population ; de mĉmc, ccrtaincs familles nucléaires (parcnt-cnfant) nc comprenaicnt que des sujets très résistants. Cettc obscrvation suggérait l'existence d'unc composantc génétique à la résistance. Cette opinion était d'ailleurs confortéc par les études che» 


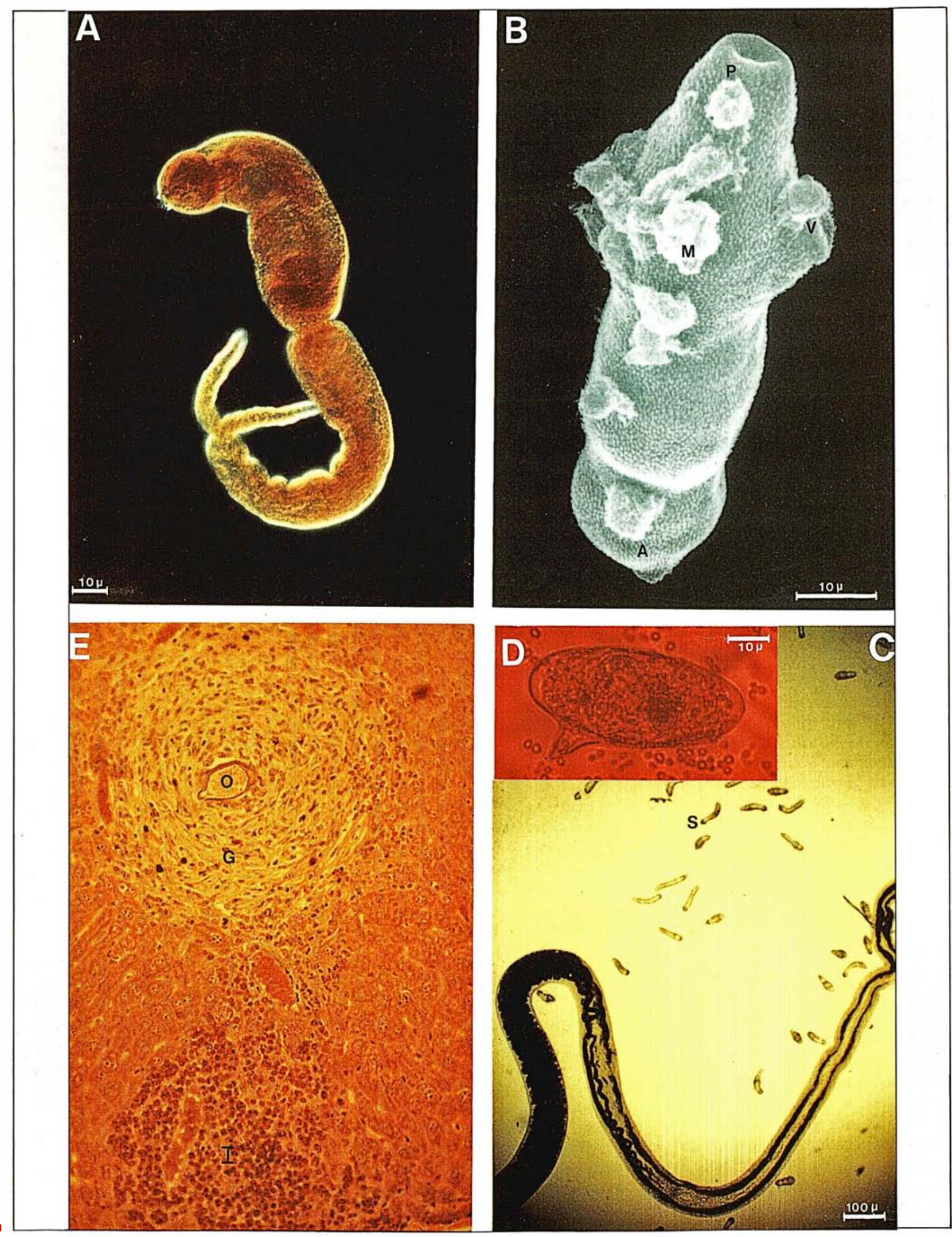


Figure 1. Le schistosome est I'agent de la bilharziose ou schistosomiase, une infection parasitaire qui affecte la santé de plusieurs centaines de millions d'individus dans le monde, en particulier dans les pays de la zone tropicale. Les schistosomes sont des organismes eucaryotes appartenant à la classe des vers plats (Trématodes) à sexe séparé. Le genre Schistosoma comprends plusieurs espèces dont les plus importantes en médecine humaine sont $\mathrm{S}$. mansoni, S. haematobium, S. japonicum et S. intercalatum; ces espèces causent chez l'homme des affections différentes, S. mansoni et S. japonicum étant les plus pathogènes. Les schistosomes développent leur cycle entre deux hôtes, I'un est un mollusque aquatique d'eau douce, l'autre est généralement l'être humain. La forme larvaire nageuse (A) émise par le mollusque pénètre la peau de l'être humain par digestion des tissus où elle se transforme en quelques heures en un organisme morphologiquement et antigéniquement différent, la schistosomule (B, avec l'autorisation de J. Caufield, Harvard University. Les monocytes (M) adhérant à la surface de la schistosomule rappellent que celle-ci est multicellulaire). Cette larve migre ensuite dans le système vasculaire et se développe en 4 à 5 semaines en un vers adulte qui s'établit dans les rameaux de la veine porte où il vit plusieurs années. La figure $C$ représente un vers femelle entouré d'une vingtaine de schistosomules (S). Dès la cinquième semaine, les adultes copulent et se reproduisent. La femelle pond des œufs (1D) qui digèrent la paroi intestinale, passent dans l'intestin et sont éliminés dans les fèces, contribuant ainsi à la propagation du parasite, car les œufs, au contact de l'eau, éclosent et libèrent une larve qui, à son tour, infecte le mollusque vecteur. Une partie de la ponte est toutefois entraînée par le courant sanguin vers les sinusoïdes hépatiques où les œufs restent bloqués et initient une réaction inflammatoire à médiation cellulaire (bas de la figure E) ; comme l'œuf $(O)$ ne peut être détruit et résorbé rapidement, l'infiltrat inflammatoire (I) s'organise en un granulome (G) dont un des rôles est d'empêcher la diffusion des substances antigéniques toxiques de l'œuf, mais qui a également pour résultat la destruction locale du tissu hépatique. Cette étape destructive est suivie par une phase de réparation ou cicatrisation durant laquelle les tissus endommagés sont remplacés par des tissus fibreux (haut de la figure E). Les infections élevées sont donc associées à une hépatite chronique (inflammation causée par les œufs et les produits de sécrétion des vers), associée et suivie par un dépôt massif de collagène autour des rameaux de la veine porte. D'un point de vue macroscopique, les rameaux portes dont les parois sont épaissies par les tissus fibreux, prennent l'aspect de tuyaux de pipe qui est caractéristique de cette fibrose que Symmers fut un des premiers à décrire (fibrose dite de "Symmers" en "tuyau de pipe "). D'un point de vue clinique, I'hépatite évolue en cirrhose dont les conséquences et complications sont, hélas, trop connues : hypertension portale, développement de varices cesophagiennes, saignements, accélération de la nécrose du tissu hépatique, ascite et mort par hémorragie et cachexie. Cinq à $10 \%$ des individus vivant en zone d'endémie développent une forme clinique grave; $V$ : ventouse; $A$ : pôle antérieur ; $P$ : pôle postérieur ; $M$ : monocyte.

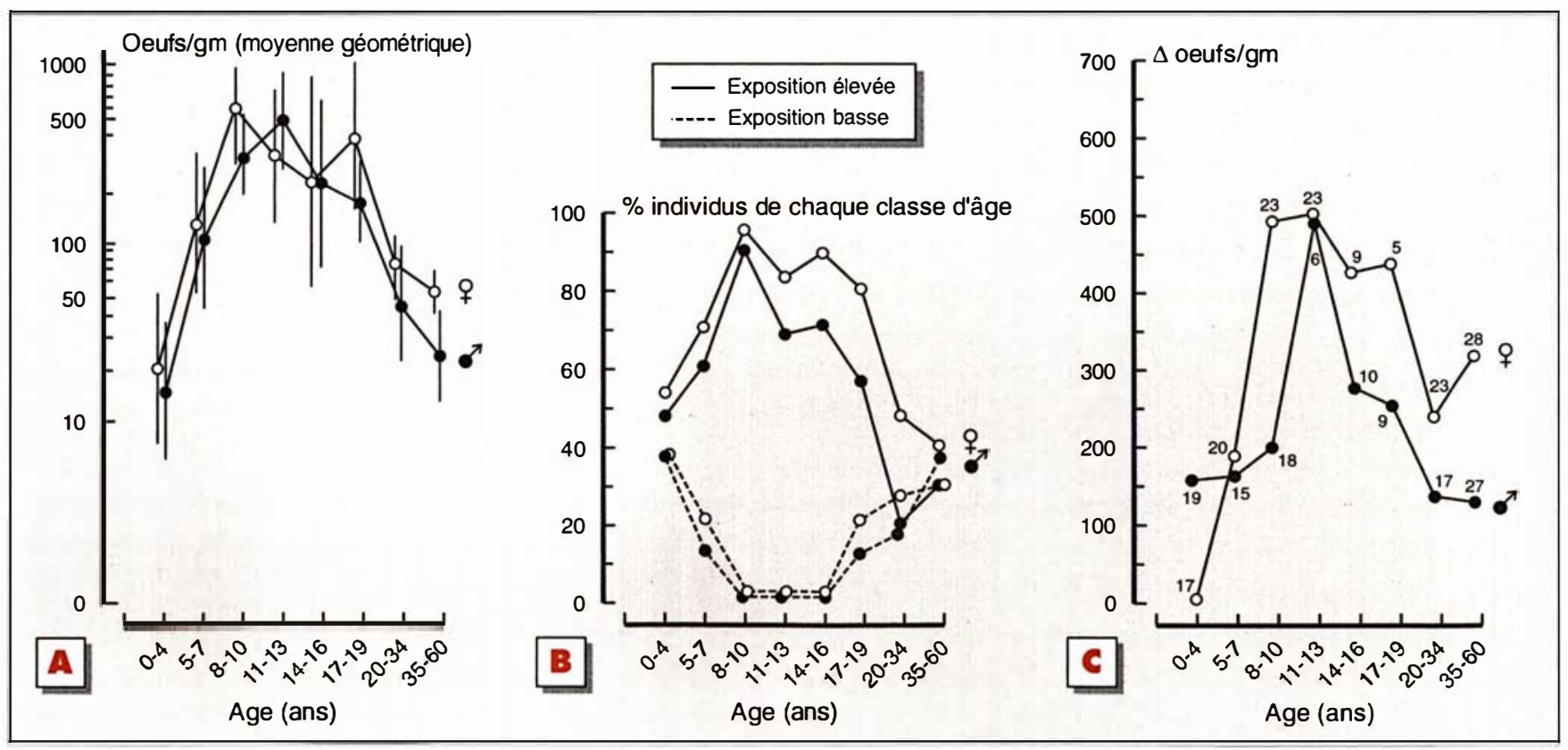

Figure 2. Niveaux d'infection pour chaque classe d'âge avant (A) et après (C) prise en compte des différences d'exposition entre les classes d'âge (B). (A) Niveau d'infection brute dans chaque classe d'âge. Les niveaux d'infection sont exprimés comme la moyenne géométrique du nombre d'œufs excrétés. (B) Variations de l'exposition en fonction de l'âge. Le pourcentage d'individus ayant une exposition élevée $(W=3+4)$ ou basse (W=1) est indiqué pour chaque classe $d^{\prime}$ âge. (C) Influence de l'âge sur les niveaux d'infection après prise en compte de l'effet de l'exposition et du sexe. Les résultats sont représentés comme la différence, en nombre d'œufs excrétés, entre chacune des classes d'âge et la classe d'âge (0-4 ans) qui présente le niveau d'infection moyen le plus bas. Les chiffres figurant au-dessus des courbes représentent le nombre d'individus par point. 


\section{ANALYSE DE SÉGRÉGATION ET ANALYSE DE LINKAGE}

L'analyse de ségrégation est la première étape nécessaire pour déterminer, à partir de données familiales, le mode de transmission d'un phénotype, avec comme but principal l'identification d'un gène unique, appelé gène majeur. Le principe général de cette méthode est de déterminer, par des tests statistiques, le mode de transmission expliquant le mieux les distributions familiales observées du phénotype étudié. Des modèles mathématiques ont récemment été développés [12, 13] et incluent l'influence de facteurs environnementaux et génétiques dans les différentes hypothèses concernant le mode de transmission du phénotype. Dans ces modèles, l'expression du phénotype est supposée être sous la dépendance de trois composantes. (a) Des facteurs de milieu. Lorsque le phénotype étudié est quantitatif, comme pour notre étude sur l'infection à S. mansoni où le phénotype était le nombre d'œufs du parasite dans les selles, la prise en compte des facteurs de milieu se fait par un ajustement des données sur ces facteurs par des méthodes statistiques classiques. On obtient alors une distribution du phénotype indépendante des facteurs de milieu avec une moyenne $\mathrm{m}$ et une variance $\mathrm{s}^{2}$. (b) Un gène majeur mendélien diallélique ( $A$ et a, par exemple). La prise en compte de ce gène majeur se fait en considérant que l'échantillon est constitué de trois groupes d'individus, correspondant aux trois génotypes possibles ( $A A$, Aa et aa). Dans ce cas, la distribution du phénotype n'est plus homogène (avec une seule moyenne $\mathrm{m}$ ), mais composée d'une combinaison de trois distributions avec trois moyennes $\mathrm{mAA}, \mathrm{mAa}$ et maa correspondant aux trois génotypes: les proportions de ces trois distributions correspondent aux fréquences respectives des trois génotypes, elles-mêmes données par la fréquence d'un des allèles (A par exemple) et par la loi de Hardy-Weinberg (Tableau I). De plus, la répartition dans l'échantillon de ces trois groupes d'individus n'est pas aléatoire, mais doit respecter dans les familles les lois de la transmission mendélienne (Tablcau II). (c) Des facteurs de corrélation familiale non spécifiques, c'est-à-dire sans hypothèse particulière sur leur origine (génétique ou environnementale). Leur principe de calcul est proche d'un calcul de corrélation classique entre deux variables quantitatives; on peut ainsi déterminer un cofficient de corrélation époux-époux, père-enfant, mère-enfant et enfant-enfant.

Les différentes hypothèses pouvant expliquer les distributions familiales observées (présence de facteurs de milieu, de facteurs de corrélation familiale non spécifiques ou d'un gène majeur) peuvent s'emboîter les unes dans les autres et être testées les unes par rapport aux autres par des méthodes statistiques classiques (rapport de vraisemblance). Schématiquement, l'analyse peut se décomposer de la façon suivante :

- ajustement des données sur les facteurs de milieu influençant significativement le phénotype étudié;

- sur les données ajustées, on répond alors aux questions suivantes.

- existe-t-il une ressemblance familiale? On teste si la présence des facteurs de corrélation familiale non spécifiques améliore significativement l'explication des observations;

- si oui, cette ressemblance est-elle, au moins en partie, expliquée par la présence d'un gène majeur? On ajoute l'effet du gène majeur aux facteurs de corrélation précédents et on teste si cet apport entraîne une amélioration significative;

- si oui, les distributions familiales observées sont-elles compatibles avec les lois de la transmission mendélienne? Différents tests permettent alors de vérifier la compatibilité des distributions observées avec celles attendues d'après les lois de Mendel.

Ce n'est qu'en cas de réponse positive à toutes ces questions que l'on pourra conclure, avec un risque minimal d'erreur, à la présence d'un gène majeur intervenant dans l'expression du phénotype étudié.

$L$ 'analyse de linkage permet de localiser le gène étudié sur le chromosome en étudiant dans les familles sa transmission conjointe avec des marqueurs génétiques. Cependant, cette méthode nécessite que le rôle de ce gène dans l'expression du phénotype étudié ait déjà été prouvé par d'autres moyens, et en particulier par une analyse de ségrégation préalable.

l'animal qui montraient un contrôle génétique de la susccptibilité à l'infection par $S$. mansoni des animaux de laboratoirc. Pour évaluer cettc hypothèse, nous ne pouvions évidemment pas utiliser les méthodes expérimentales (contrôle des croiscments et de l'infection, lignécs congéniques). Les moyens disponibles pour mettre en évidence l'intervention de facteurs génćtiques dans des affections multifactoriclles, comme les maladics infecticuses, sont des méthodes statistiques [11], essenticllement l'analyse de sćgrégation et l'analyse de linkage (voir encart, ci-dessus).

Pour identifier les factcurs génétiques impliqués dans le contrôle de la susceptibilité à l'infection par $S$. mansoni entrepris unc analysc de ségrégation sur des donnćes recueillies à partir dc 45 familles.

La premic̀re étape de cettc analyse cst l'ajustement des données brutes (nivcaux d'infection) sur les factcurs dont nous avions pu démontrer l'influence sur l'infection (exposition au parasitc, âge et sexe des individus). L'ajustement global sur ces trois factcurs a été effectué par régression multivarićc.

L'analysc de sćgrégation a ćté réalisćc sur les phénotypes ajustés et en utilisant les deux modèles récemment décrits, lc modèle mixtc unifié et le modèle régressif $([12,13]$ et encart cidessus). Ces modèles ont deux différences cssentielles : d'unc part, ils ne caractérisent pas les corrélations fami- liales non spécifiques de la même façon; d'autre part, le modèlc régressif permet de considérer des grandes généalogics dans lcur enscmblc, alors que lc modèlc mixte unifié nécessitc un découpage préalable dc ccs généalogies en lcurs familles nucléaircs (parcnt-cnfant) constitutives.

Les résultats obtenus en analysant les donnćcs sclon ces dcux modèles sont similaires. Il y a unc indication très clairc en favcur d'un gène majcur contrôlant l'intensité de l'infection par S. mansoni, les distributions obscrvécs ćtant compatibles avec les lois de la transmission mendéliennc. Les estimations des paramètres de ce gènc majcur sont ćgalement proches avec les dcux modèles utilisés. 


\begin{tabular}{l} 
Tableau I \\
LOI DE HARDY-WEINBERG \\
$\begin{array}{l}\text { Cette loi stipule que dans une population sans sélection ni mutation et où } \\
\text { les mariages se font au hasard (panmixie), les fréquences à l'origine, } q \text { et } \\
1-q \text {, des allèles } A \text { et a d'un gène diallélique se maintiennent de génération } \\
\text { en génération de même que la fréquence des génotypes associés AA, Aa } \\
\text { et aa avec les valeurs données dans le tableau }\end{array}$ \\
\hline \\
\cline { 2 - 7 }
\end{tabular}

La fréquence de l'allèle de susceptibilité (A) est estimée entre 0,20 et 0,25 . Il y a donc, selon la loi de HardyWeinberg (expliquée dans le Tableau I), environ $5 \%$ d'homozygotes AA susceptibles, $60 \%$ d'homozygotes aa résistants, et $35 \%$ d'hétérozygotes Aa. La distribution globale du phénotype en présence d'un gène majeur est présentée dans la figure 3 , p. 114. Le niveau de susceptibilité des hétćrozygotes Aa est assez proche de celui des homozygotes résistants aa. Cependant, l'hypothèse d'un gène purement récessif (Aa et aa confondus) n'était pas compatible avec les données. Le génotype le plus probable des membres de cinq familles nucléaires, déterminé à partir de leur niveau d'infection ajusté pour l'effet de l'exposition (W), de l'âge (Y) et du sexe, est représenté sur la figure 4 (p. 115).

Au total, l'ensemble des analyses permettent de conclure à la présence d'un l'homme, la susceptibilité à l'infection par S. mansoni. Les résultats obtenus avec deux modèles différents sont tout à fait concordants. Une analyse de linkage doit être maintenant effectuée pour confirmer l'existence de ce gène et le localiser dans le génome humain. Les principales conclusions des études épidémiologiques et génétiques sont ainsi les suivantes : (1) les variations inter-individuelles des niveaux d'infection peuvent être expliquées en partic seulement (20 à $25 \%$ de la variance) par les effets de facteurs de milieu (ćcologiques et comportementaux) alors que des facteurs génétiques sont à l'origine de 30 à $40 \%$ de la variance; (2) le poids des facteurs de résistance (âge et gène majeur) est très important puisque leurs effets expliquent plus de $50 \%$ de la variance des niveaux d'infection. Ce résultat montre que priorité doit être donnée aux gène majeur contrôlant, chez

\section{Tableau II}

\section{LOIS DE LA TRANSMISSION MENDÉLIENNE}

Le tableau donne la probabilité des génotypes d'un enfant conditionnellement à ceux de son père, $\mathrm{Gp}$, et de sa mère, $\mathrm{Gm}$. Les valeurs entre parenthèses donnent les probabilités correspondant aux génotypes $A A$, $A a$ et aa, respectivement

\begin{tabular}{|c|c|c|c|}
\hline & & Gp & \\
\hline $\mathrm{Gm}$ & AA & $\mathbf{A a}$ & aa \\
\hline $\begin{array}{l}\mathrm{AA} \\
\mathrm{Aa} \\
\text { aa }\end{array}$ & 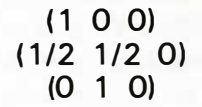 & 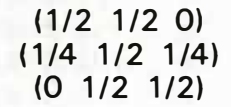 & $\begin{array}{c}\left(\begin{array}{lll}(0 & 1 & 0\end{array}\right) \\
\left(\begin{array}{ccc}0 & 1 / 2 & 1 / 2\end{array}\right) \\
\left(\begin{array}{lll}0 & 0 & 1\end{array}\right)\end{array}$ \\
\hline
\end{tabular}

$m / s \quad n^{\circ} 2$, vol. 8 , févier 92

méthodes de contrôle, telle la vaccination, qui visent à augmenter les capacités de résistance ; (3) le contrôle de la résistance par un gène majeur suggère que les bas niveaux de résistance pourraient résulter d'altérations affectant une même fonction; (4) les mécanismes de résistance se mettent en place lentement chez les enfants et les adolescents, probablement parce que ces mécanismes requièrent pour leur développement une exposition prolongée au parasite et de multiples réinfections. Ce processus est altéré chez les sujets très exposés à l'infecetion.

\section{Quelle place accorder aux mécanismes d'immunité acquise dans la résistance ?}

Des travaux (résumés dans [14]) conduits chez le rat, la souris et le singe montrent qu'une infection par des cercaires intactes ou irradiées, et que l'injection d'extraits ou d'antigènes parasitaires confèrent une certaine protection à ces animaux contre une surinfection par des cercaires. Les anticorps jouent un rôle important dans les mécanismes de protection, en particulier chez le rat et, dans une moindre mesure, chez la souris. Ceux-ci interviendraient dans la destruction du parasite, soit en activant le système du complément, soit en intervenant dans les réactions de cytotoxicité cellulaire (monocytes/macrophages et ćosinophiles). Plusieurs études ont également muntré la rapide transformation de la larve en un organisme réfractaire à la détection et à la destruction par le système immunitaire, soulignant ainsi l'importance d'une mobilisation rapide des réactions de défense au site de pénétration du parasite. Chez le rat, et probablement chez le singe, cette mobilisation s'effectue en grande partie par les médiateurs secrétés par les mastocytes. Ces cellules sont armées par les $\operatorname{IgE}$, le pontage des $\operatorname{IgE}$ par les antigènes larvaires stimule ces cellules, provoquant la sécrétion par celles-ci de puissants médiateurs de la réponse inflammatoire. En fait, le rôle des $\operatorname{IgE}$ dans les mécanismes de défense contre les infections par les helminthes ne se limite probablement pas à leurs propriétés sensibilisantes 
pour les mastocytes de la peau : ces infections sont en effet caractérisées par des taux d'IgE très élevés, 100 à 1000 fois plus élevés que les taux observés dans d'autres infections et ces immunoglobulines interviennent efficacement dans la destruction des larves d'helminthes par les monocytes, les plaquettes et les éosinophiles [15-17] (dont le nombre dans le sang augmente considérablement dans ces infections). La résistance des rats à l'infection par certains helminthes, dont $S$. mansoni, est augmentée par le transfert passif de sérums immuns enrichis en $\operatorname{IgE}[18,19]$ et est diminuée par la suppression néonatale de la synthèse d'IgE [20, 21].

Ces observations nous ont naturellement conduits à évaluer la réponse $\operatorname{IgE}$ chez les sujets très résistants et à comparer les résultats obtenus avec les observations effectuées chez les individus sensibles (figure 5; p. 116) : les taux sériques des IgE antilarvaires se sont avérés être, en moyenne, six à huit fois plus élevés dans le groupe résistant que dans le groupe sensible. Non seulement les sérums des sujets sensibles contenaient moins d'IgE anti-larvaires, mais encore ils renfermaient des taux élevés d'anti- corps capables d'entrer très efficacement en compétition avec les $\operatorname{IgE}$ pour la fixation de l'antigène. Ce phénomène est illustré sur la figure $6 A, p .117$, qui montre l'augmentation considérable du signal de détection des allergènes larvaires lorsque les $\operatorname{IgE}$ sont purifiées ๑ avant d'être utilisées pour révéler les antigènes transférés sur filtre (comparer avec le signal obtenu avec les sérums non fractionnés $\Theta$ ). Ces anticorps compétiteurs ont également été observés dans les sérums de sujets infectés par des filaires et sont bien connus des allergologues, car ils sont produits par les individus atopiques en cours de désensibilisation.

Ces observations nous ont amenés à prendre en compte l'effet des facteurs compétiteurs lors de l'évaluation des IgE dans la résistance. Cela fut l'objet d'une étude plus vaste, élargie à des sujets appartenant à toutes les classes d'âge et d'exposition. Tous les isotypes (excepté les $\operatorname{IgA}$ ) ont été évalués simultanément comme variables explicatives des niveaux de réinfection après traitement. Le résultat de ces études multivariées (" multivariées " car elles évaluent simultanément plusieurs variables, par opposiMed Hyg 1985 ; 79 : 393-408.

5. Wilkins HA. Reinfection after treatment of schistosome infections. Parasitol Today $1989 ; 5: 83-7$.

6. Anderson RM, Mayer M. Herd immunity to helminth infections and implications for parasite control. Nature 1985; 315 : 493-6.

7. Clarke V. The influence of acquired resistance in the epidemiology of bilharziasis. Centr Afr J Med 1966 ; 12 (suppl) : 1-30.

8. Kloetzl K, Da Silva JR. Schistosomiasis mansoni acquired in adulthood : behaviour of egg counts and the intradermal test. $A m \mathrm{~J}$ Trop Med Hyg 1967; $16: 167-9$.

9. Butterworth AE, Bensted-Smith $R$ Capron $\mathrm{A}$, et al. Immunity in human schistosomiasis mansoni : prevention by blocking antibodies of the expression of immunity in young children. Parasitology 1987 ; 94 . 281-300.

10. Rihet $\mathrm{P}$, Demeure $\mathrm{CE}$, Bourgois $\mathrm{A}$, Prata A, Dessein AJ. Evidence for an association between human resistance to Sichistosoma mansoni and high anti-larval $\mathrm{IgE}$ levels. Eur J Immunol 1991 ; 2679-86.

11. Demenais F, Bonati C. Analyse de ségrégation, évolution des méthodes d'analyse. In : Feingold $\mathrm{J}$, ed. Génétique

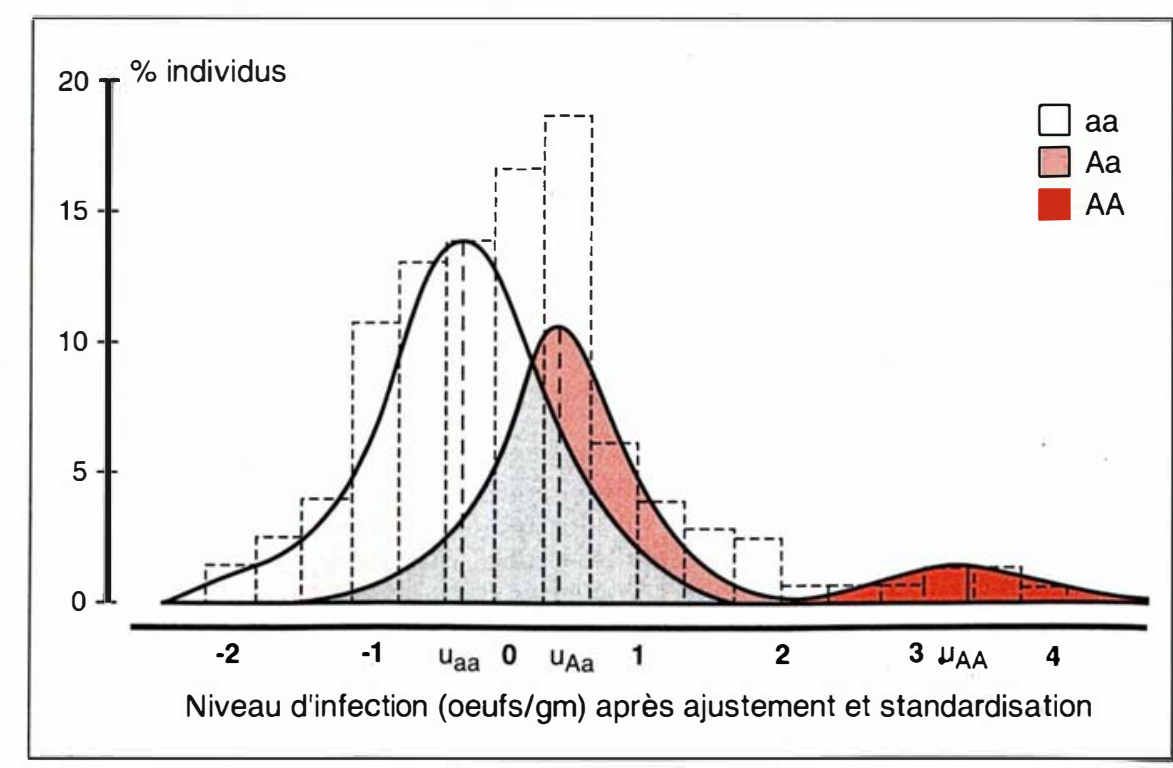

Figure 3. Distribution du phénotype "intensité d'infection " telle qu'elle est prédite par le modèle du gène co-dominant majeur, en supposant une distribution normale pour chaque génotype. L'histogramme représente la distribution des niveaux d'infection après ajustement et standardisation ; $A$ : allèle de susceptibilité. 


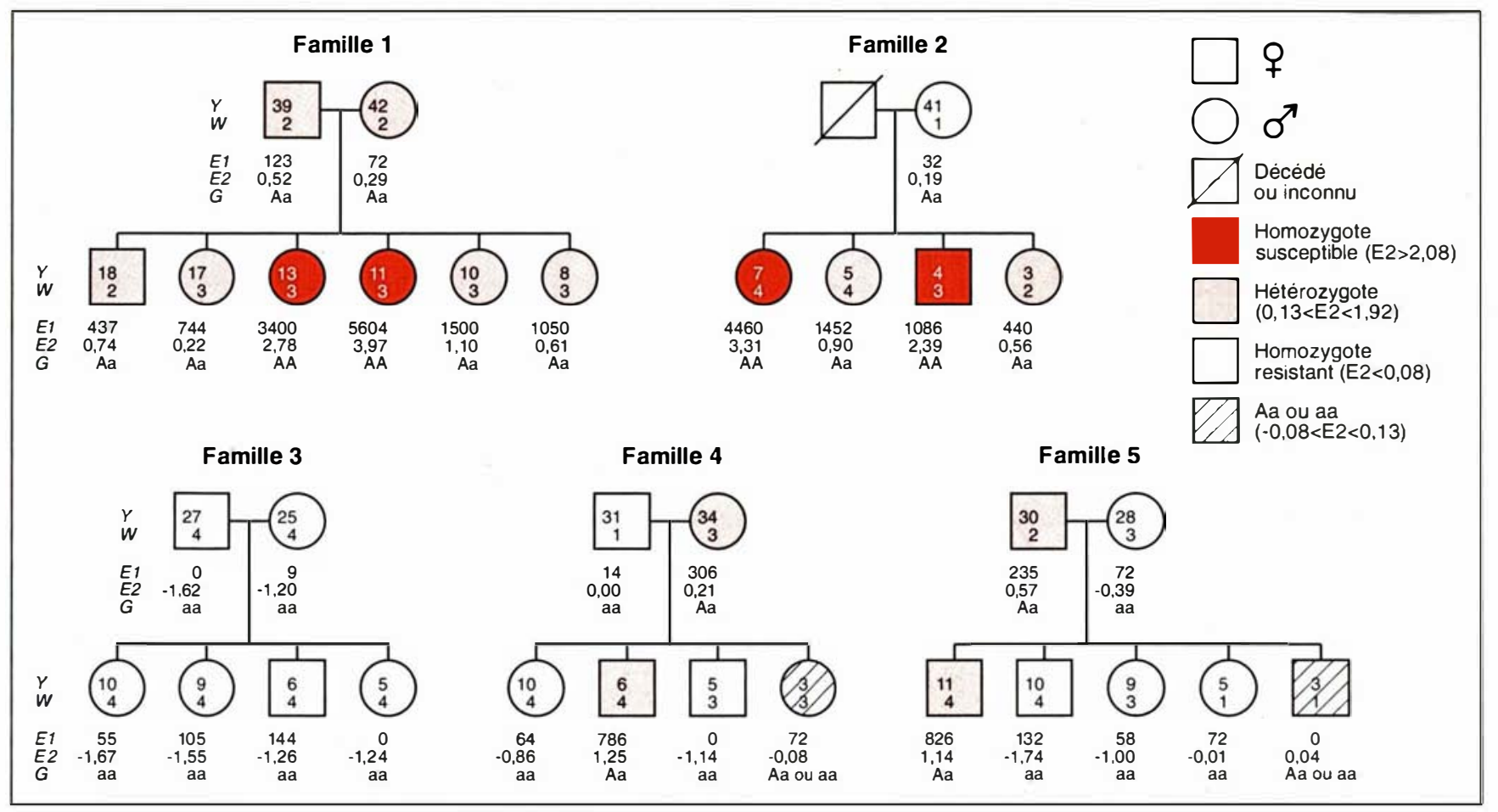

Figure 4. Age (Y) niveau d'exposition (W), niveau d'infection bruts (E1) et ajustés (E2) sur $Y$, W, le sexe et I'âge pour les membres de cinq familles. Le génotype $G$ de chaque individu, dans le modèle du gène co-dominant majeur, est également indiqué.

tion aux analyses " univariées" qui étudient chaque variable successivement) confirma que les hauts niveaux de résistance sont associés à des taux d'IgE élevés. Mais cette association ne put être démontrée que lorsque les IgG4 (et seulement les $\operatorname{IgG} 4$ ) furent également incluses dans l'analyse statistique comme variable explicative ayant une association négative à la résistance. Ces résultats suggèrent que les IgE et les IgG4 exercent sur les capacités de résistance des effets antagonistes, positifs pour les IgE et négatifs pour les $\operatorname{IgG} 4$. La résultante de ces effets protecteurs des $\operatorname{IgE}$ et anti-protecteurs des IgG4 déterminerait, en partie, les niveaux de résistance. Des résultats comparables ont été obtenus par P. Hagan et al. [22] dans leur étude en Gambie sur des sujets infectés par l'agent de la bilharziose urinaire ( $S$. haematobium). De récentes observations de Butterworth et al., effectuées au Kenya chez des sujets infectés par $S$. mansoni, vont également dans le même sens (But$m / s n^{\circ} 2$, vol. 8, février 92 terworth et al., manuscrit soumis pour publication).

Ces études indiquent aussi que le lent développement de la résistance avec l'âge, et son altération chez les adolescents très exposés, peuvent s'expliquer par les fluctuations de l'équilibre IgE/IgG4. En particulier, la forte exposition des jeunes adolescents produirait un déséquilibre de ce rapport en faveur des IgG4 qui, à la différence des IgE, augmentent avec l'exposition. L'effet anti-protecteur des IgG4 n'est pas complètement expliqué ; toutef ois, de récents travaux ont montré que les IgG4 : (1) exercent un puissant effet compétiteur sur les $\operatorname{IgE}$ dans la fixation de ceux-ci à l'antigène et inhibent l'activation spécifique des basophiles (Demeure et Dessein, manuscrit en préparation) et (2) pourraient bloquer certaines réactions d'ADCC (cytotoxicité dépendante des anticorps) [23].

Nous ignorons actuellement si le gène co-dominant majeur contrôle le rapport $\mathrm{IgE} / \mathrm{IgG} 4$ ou si ses effets sont indépendants de celui-ci. Cette der- nière possibilité n'est pas exclue car d'autres facteurs immunologiques, indépendants des $\operatorname{IgE}$ et $\operatorname{IgG} 4$, ont probablement une influence importante sur les niveaux de résistance. Ainsi, notre laboratoire et celui d'A. Butterworth [24] ont montré que les IgG2, dirigées essentiellement contre des déterminants sucrés, pourraient avoir un effet facilitant sur la réinfection, effet qui serait indépendant de l'âge et de l'effet des IgE et des IgG4. Cette dernière observation illustre la multiplicité des mécanismes qui interviennent pour déterminer les niveaux de résistance de l'être humain à S. mansoni. Parmi ces mécanismes, certains pourraient être contrôlés par le gène co-dominant majeur.

\section{Vacciner contre les schistosomes}

Parmi les moyens qui peuvent être utilisés pour augmenter la résistance des sujets vivant en zone endémique, 


\section{RÉFÉRENCES}

12. Lalouel JM, Rao I)C, Morton NE, Elston RC. A unified model for complex segregation analysis. Am J Hum (ienet $1987 ; 35$ : $816-26$.

13. Bonney GE. On the statistical determination of major gene mechanisms in continuous human traits : regressive models. $A m$ J Med Genet 1984; 18: 731-49.

14. Vignali I)AA, Bickle (2I), 'Taylor MG. Immunity to Schistosoma mansoni in vivo : contradiction or clarification. Immunol Today $1989 ; 10: 410-6$.

15. Capron A, Dessaint JP, Capron M, Bazin II. Specific IgE antibodies in immune adherence of normal macrophages to Schistosoma mansoni schistosomules. Nature 1975 ; $253: 474-5$

16. Capron M, Bazin II, Joseph $M$, Capron A. Evidence for IgE-dependent cytotoxicity by rat eosinophils. J Immunol 1981; $126): 1764-8$

17. Joseph M, Auriault C, Capron A, Vorng M, Viens P. A new function for platelets : IgE dependent killing of schistosomula. Nature $1983 ; 303: 810-2$

18. Ogilvie BM, Smithers SR, Terry RJ. Reagin-like antibodies in experimental infections of Schistosuma mansoni and the passive transfer of resistance. Nature $1966 ; 209$ $1221 \cdot 3$

19. ('apron A, Dessaint JP, Capron M, Joseph M, Pestel J. Role of anaphylactic antibodies in immunity to schistosomes. $A m$ J Trop Med Hlys, 1980); 29: 849-57.

20. Kigoni EP, klsas PPX, Lenzi HL, Dessein AI. IgE antibody and resistance to infection. II. Effect of IgE suppression on the early and late skin reaction and resistance to Schistosoma mansoni infection. Eur J Immunol 1986 ; 16 : 589-95.

21. Dessein AJ, Parker WI, James SL, 1)avid JR. IgL antibody and resistance to infection. Selective suppression of the $\operatorname{IgE}$ antibody response in rats diminishes the resistance and the eosinophil response to Trichinella spiralis inlection. I Exp Med 1981; 153: 423-36.

22. Hagan $P$, Blumenthal UJ, Dunn 1), Simpson AJG, Wilkins HA, Human IgE, IgG4 and resistance to reinfection with Schistosoma huematobium. Nature $1991 ; 349$ : 243-5.

23. Khalife JI), Dunne I)W, Richardson BA. et al. Functional role of human IgG subclasses in eosinophile mediated killing of schistosomula of S. mansoni. J Immunol 1989 ;
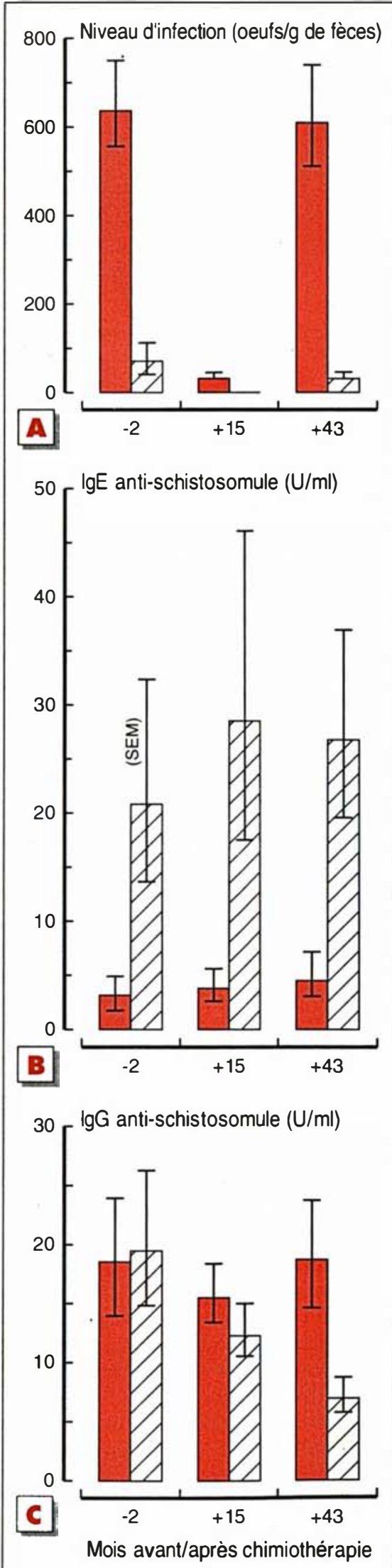

Sujets sensibles $\square$ sujets résistants deux sont privilégiés : vaccination et chimiothérapie. Les effets de la chimiothérapie sur les niveaux de résistance ont été encore insuffisamment explorés; il semble toutefois que le traitement par des agents schistosomicides pourrait augmenter la résistance, mais de manière transitoire seulement. La vaccination est sans aucun doute la méthode de contrôle la mieux adaptée aux conditions économiques et au système de santé des pays où se rencontrent les bilharzioses. Le vaccin peut être peu coûteux si les méthodes de génie génétique sont utilisées pour produire les antigènes vaccinants ; l'utilisation de ces techniques est d'ailleurs requise par la nécessité de débarrasser le vaccin des constituants parasitaires qui sont toxiques, tolérogéniques ou pathogéniques. Ces antigènes pourraient être intégrés à un vaccin multivalent dirigé contre plusieurs agents infectieux : ainsi sa distribution ne nécessiterait pas de campagnes de vaccination particulières, d'autant que les vaccins sous-unitaires (constitués d'antigènes purifiés) sont plus stables que les vaccins constitués de parasi-

Figure 5. Les niveaux sériques des IgE antilarvaires (B) sont augmentés chez les sujets les plus résistants par comparaison à des sujets moins résistants. En revanche, les niveaux des IgG (C) et IgM sont, en général, comparables entre ces deux groupes et augmentent chez les sujets se réinfectant massivement. Les jeunes sujets de cette étude ont été divisés en deux groupes sur la base de leur niveau de résistance à l'infection avant et après déparasitage complet par l'oxamniquine (A). Tous les sujets avaient un niveau d'exposition très élevé durant les 46 mois de cette étude. Certains $(n=20)$ étaient très infectés avant traitement, se réinfectèrent dans les 15 mois après traitement et développèrent des niveaux d'infection très élevés 43 mois après traitement. IIs furent considérés comme les plus susceptibles. Les sujets $(n=18$ ) peu ou pas infectés, malgré un haut niveau $d^{\prime}$ exposition, résistèrent à la réinfection ou développèrent de faibles infections après traitement. Ils furent considérés comme les plus résistants. 


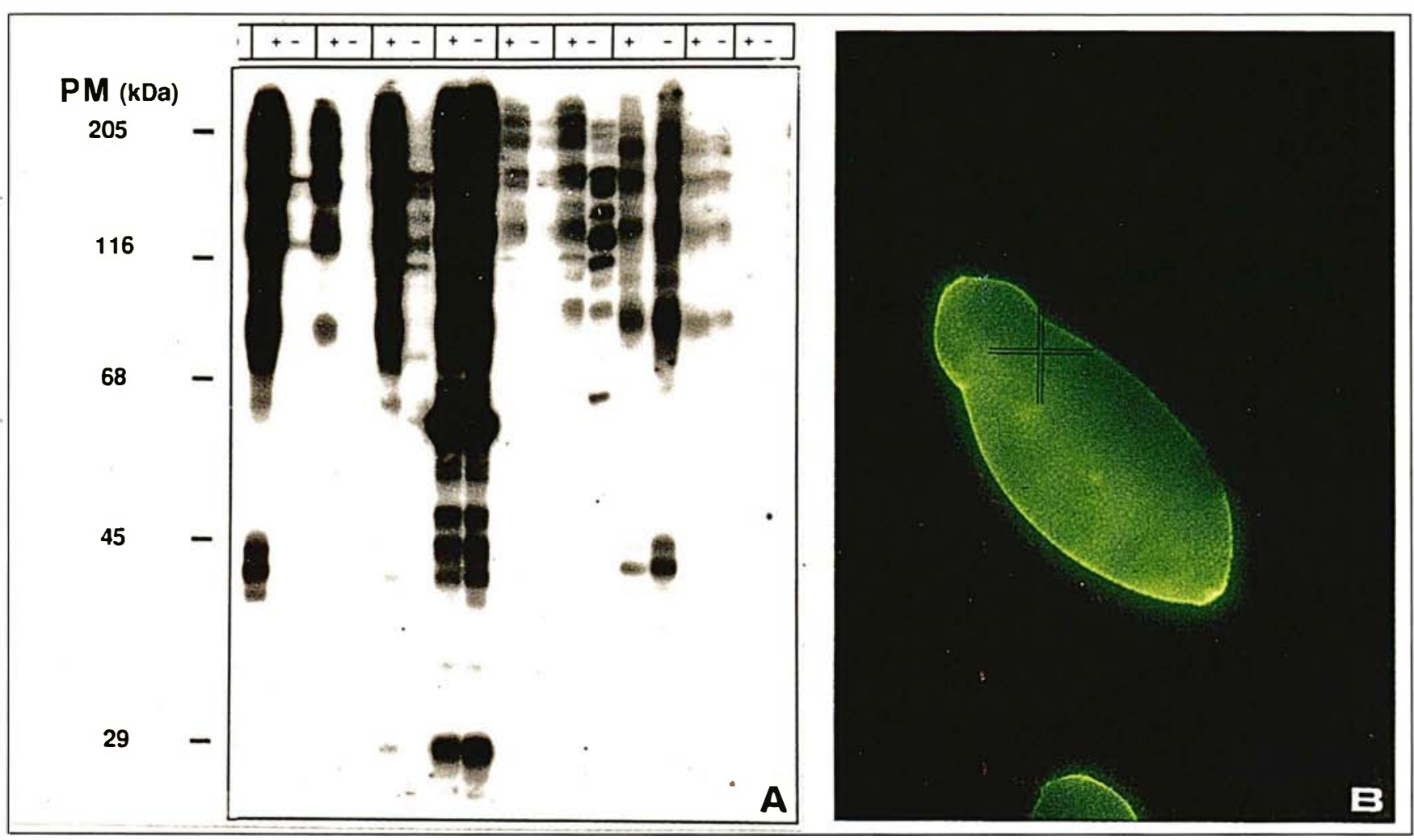

Figure 6. Mise en évidence de molécules allergéniques dans les extraits membranaires (A) et à la surface des larves (B). A. Les extraits membranaires de larves ont été fractionnés par électrophorèse en gel d'acrylamide et transférés sur des filtres de nitrocellulose; ces filtres ont été découpés en bandes et celles-ci ont été incubées, soit avec des sérums (-) prélevés sur les sujets étudiés, soit avec les fractions enrichies en IgE de ces sérums (+). Les IgE qui se sont fixées au filtre sont révélées avec une sonde spécifique des IgE et marquée à l'iode 125. B. Les larves vivantes ont été incubées avec des IgE purifiées des mêmes sérums; après lavage, les IgE fixées à la surface des larves ont été révélées avec une sonde fluorescente spécifique des lgE.

tes atténués ou tués, lesquels requièrent souvent une chaîne du froid.

Toutefois, la mise au point d'un vaccin anti-schistosome n'est pas une entreprise facile. Il faut se rappeler que les vaccins efficaces, disponibles en médecine humaine, protègent contre des agents infectieux bactériens ou viraux qui, en général, induisent un haut niveau de protection chez les individus qu'ils infectent [25], alors que les infections par les schistosomes, même répétées et traitées, ne stimulent pas une forte immunité protectrice. Cela est la conséquence de l'adaptation remarquable des parasites à leurs hôtes, laquelle leur permet d'échapper aux réactions du système immunitaire de celui-ci par toute une série de subterfuges tels la variation antigénique, le mimétisme $m / s n^{\circ} 2$, vol. 8 , février 92 moléculaire ou l'induction d'états de paralysie immunologique. La sophistication de ces mécanismes d'échappement est à mettre en parallèle avec la possession par les parasites, organismes eucaryotes, d'un génome beaucoup plus important que celui des agents infectieux procaryotes.

Malgré ces difficultés, plusieurs antigènes capables d'induire une protection incomplète mais réelle chez l'animal ont été identifiés et représentent une étape importante sur la voie du vaccin [26]. Certains de ces antigènes pourraient entrer bientôt en phase d'expérimentation chez l'être humain. L'objet de cet article n'est pas de détailler ces excellents travaux et le lecteur en trouvera une description concise dans de récentes revues [27-29].
En parallèle à ces études chez l'animal, les cibles de la réponse immune des sujets résistants à l'infection ont été également étudiées : la résistance a été associée à la production d'anticorps dirigés contre une protéine détectable à la surface de la larve [2]. L'analyse de la séquence en acides aminés de cet antigène révéla de fortes homologies (72\%) avec la séquence d'une enzyme de la glycolyse, la glycéraldéhyde-3-Pdéshydrogénase (GADPH) [30]. Tous les acides aminés impliqués dans le site catalytique de l'enzyme sont conservés dans la molécule recombinante, indiquant que, très probablement, cet antigène possède l'activité catalytique GAPDH. D'autres cibles importantes de la réponse immune, en particulier certains antigènes pro- 
tecteurs identifiés chez l'animal, présentent de fortes analogies avec des enzymes telles que la triose-Pisomérase [31] et la glutathione-Stransférase [32, 33]. Il est possible que ces enzymes, normalement cytosoliques, passent en surface du parasite au niveau des lésions qui sont infligées au tégument par le système immunitaire. Il est également possible que la GAPDH et la triose-Pisomérase, qui sont normalement étroitement associées à l'actine, viennent en surface lorsque l'actine fait protrusion dans les lésions du tégument, où clle pourrait organiser le processus de réparation [34].

La découverte de molécules fortement conservées à la surface du parasite n'est pas surprenante : ce résultat est en accord avec la théorie du mimétisme moléculaire suivant laquelle le parasite se couvre de molécules semblables à celles de son hôte pour échapper à la reconnaissance par les systèmes effecteurs de celui-ci [35]. Ce qui est plus inattendu, c'est que ces molécules soient des antigènes majeurs, cibles de la réponse anticorps. Il est possible que la conservation de ces antigènes soit à l'origine d'une restriction de leur immunogénicité à certains haplotypes du complexe majeur d'histocompatibilité (HLA) comme semblent l'indiquer les études sur l'immunogénicité de P-37 chez la souris. Une telle restriction compliquerait leur utilisation en vaccination ; cette difficulté pourrait être néanmoins résolue en incluant plusieurs antigènes dans un même vaccin, en particulier des antigènes portant des épitopes " universels " qui peuvent être présentés par tous les haplotypes HLA présents dans les populations à vacciner.

Les résultats obtenus dans l'étude de la réponse $\operatorname{Ig} E$ plaident fortement pour l'inclusion, dans le vaccin, d'allergènes majeurs de la larve, en particulier ceux des allergènes présents en surface du parasite (figure 6). Peut-on mettre à profit les propriétés des $\operatorname{IgE}$ sans courir le risque de déclencher les réactions secondaires néfastes qui sont parfois si difficiles à contrôler chez les sujets atopiques? L'absence de réactions allergiques chez les sujets vivant en zone d'endémie et résistant à l'infection suggère que cet objectif peut être atteint ; comment y parvenir n'est, toutefois, pas encore écrit

\section{Summary}

Factors controlling resistance to schistosomiasis in endemic area

In endemic area of schistosomiasis mansoni, environmental factors account for 20 to $25 \%$ of infection variance while 30 to $40 \%$ of it are accounted for by the effects of a major co-dominant gene. Furthermore, resistance develops slowly in children and is altered in children and young adolescents with high water contacts. These factors, major gene and age, that act on resistance account for more than half of the variance of infection intensities indicating that control programmes must give a priority to measures aimed at increasing resistance. Immunological studies carried out in Brazil, in Kenya and in Gambia all suggest that the variations of resistance in children and adolescents could result from the slow development of a protective immunity mediated by $\operatorname{Ig} E$ and antagonized by the blocking and competing action of IgG4. Though potent schistosomicids are available, prophylaxis, especially vaccination, is better suited for the control of this endemy. Antigens were identified in the analysis of the antibody response of resistant subjects and their vaccinating properties are evaluated. The implications of these findings for the control of schistosomiasis are discussed.

\section{TIRÉS A PART}

A. Desscin.
35. Damian RT. Common antigens wcen adult Schistosoma mansoni and the laboratory mousc. J Parasitol 1967 ; 53 : 60-4.
34. Matsumoto $Y$, Perry G, Levinc RJC, Blanton R, Mammoud A, Aikawa M ments. Nature 1988 ; 333 : 76-8.

33. Taylor JB, Vidal A, Torpier G, et al. The glutathione transferasc activity and tis sue distribution of a cloned $\mathrm{Mr} 28 \mathrm{~K}$ protective antigen of Schistosoma mansoni. EMBO J $1988 ; 7: 465$

$$
m / s n^{\circ} 2, \text { vol. 8, février } 92
$$

